\title{
Recent Trends in Non-Surgical Periodontal Care for the General Dentist - A Review
}

\begin{abstract}
R Arpita ${ }^{1}$, JL Swetha ${ }^{2}, M_{R}$ Babu $^{3}$, Rudhir $^{4}$
Abstract

The management of periodontal defects has been an ongoing challenge in clinical periodontics. In the recent past, attention has been focused more on regenerative and reconstructive therapies i.e. bone grafts, guided tissue regeneration, root conditioning, polypeptide growth factors, rather than on respective therapies. These therapeutic measures are shown to be limited in the predictability of healing and regenerative response in the modern clinical practice because oral environment presents several complicating factors that border regeneration. The 21st century appears to represent a time in history when there is a convergence between clinical dentistry and medicine, human genetics, developmental and molecular biology, biotechnology, bioengineering, and bioinformatics, resulting in the emergence of novel regenerative therapeutic approaches and focusing mainly on non surgical modalities.
\end{abstract}

The purpose of this article is to provide a review of the various non surgical therapies in use today. Future direction in this ever-changing field is also discussed. Techniques currently in use are reviewed and evaluated. They include Probiotics, Ozone therapy, Photodynamic therapy, Gene therapy, RNA interference, Nanotechnology, Perioprotect, TIPS \& BOST.

Keywords: Periodontics, oral environment, non-surgical therapy, probiotics.

\section{Introduction}

Advances in periodontal science and practice over the last decade have radically changed the understanding of periodontal diseases and have opened new, exciting prospects for both non-surgical and surgical therapy of periodontal diseases. Mechanical methods of subgingival debridement accomplished by thorough

1. Dr. Ramisetti Arpita BDS, MDS, Fellow In Oral Implantology(BOC), Associate Professor, Department of Periodontics, Mamata Dental College, Khammam, Andhra Pradesh, India.

2. Dr. Jonnalagadda Laxmi Swetha BDS, Senior Lecturer, Department of Periodontics, Lenora Institute of Dental sciences, Andhra Pradesh, India.

3. Dr. Muttineni Ramesh Babu BDS, MDS, Reader, Department of Periodontics, Mamata Dental College, Khammam, Andhra Pradesh, India.

4. Dr. Ramisetti Sudhir BDS, MDS, Reader, Department of Oral and Maxillofacial Surgery, Mamata Dental College, Khammam, Andhra Pradesh, India.

\section{Address of Correspondence:}

Dr. Ramisetti Arpita BDS, MDS, Fellow In Oral Implantology(BOC), Associate Professor, Department of Periodontics, Mamata Dental College, Khammam, Andhra Pradesh, India.

E-mail: arpita_paul@hotmail.com scaling and root planing, accompanied by oral hygiene procedures, have served as the gold standard of periodontal therapy for decades.

Current research is presently on-going to evaluate modified manual, ultrasonic, and sonic tip designs; combined treatments using ultrasonic/sonic with subgingival chemotherapeutics; and regenerative periodontal surgery, microsurgery, photodynamic therapy, tissue engineering.

\section{Probiotics}

The World Health Organization (WHO) has defined Probiotics as live organisms, which, when administered in adequate amounts, confer a health benefit on the host. Probiotics repopulate beneficial bacteria which can help to kill the pathogenic bacteria and fight against infection. They are also called "friendly bacteria" or "good bacteria". 1 Probiotics (Gibson GR \& Roberfroid MB, 1995) is defined as "non-absorbable food components that beneficially stimulate one or more of the beneficial microbe groups and thus have a positive effect on human health". Similarly, Synbiotics (Harish $\mathrm{K} \&$ Varghese $\mathrm{T}, 2006)^{2}$ has been defined as "combined administration of specific prebiotics with probiotics to provide definite health benefits by synergistic action".

Probiotics can be bacteria, moulds, yeast. But most probiotics are bacteria. Among bacteria, lactic acid bacteria are more popular. Lactobacillus acidophilus, L. casei, L. lactis, L. salivarius, L. plantrum, L. bulgaricus, 
L. rhamnosus, L. reuteri, Streptococcus thermophilus, E. faecalis, Bifidobacterium bifidum are commonly used bacterial probiotics. Probiotics can be in powder form, liquid form, gel, paste, granules or available in the form of capsules, sachets, etc. ${ }^{3}$

The physiological effects attributed to Probiotic bacteria include4- (1) reduction of gut $\mathrm{PH}$, (2) Production of antibacterial substances, e.g., organic acids, bacteriocins, hydrogen peroxide, diacetyl acetaldehyde, lactoperoxidase system, lactones, and other unidentified substances, (3) reconstruction of normal intestinal microflora after disorders caused by diarrhoea, antibiotic therapy, and radiotherapy, ${ }^{4}$ reduction of cholesterol level in the blood snd stimulation of immune functions.

\section{Probiotics and Periodontal Health}

Probiotics lower the $\mathrm{pH}$ in the oral cavity so that Pathogenic bacteria cannot form dental plaque and calculus that causes the periodontal disease. They make an excellent maintenance product because they produce antioxidants. Antioxidants prevent plaque formation by neutralizing the free electrons that are needed for the mineral formation. Probiotics are able to breakdown putrescence odours by fixating on the toxic gases (volatile sulphur compounds) and changing them to gases needed for metabolism.

Lactobacilli can produce different antimicrobial components including organic acids, hydrogen peroxide, low molecular weight antimicrobial substances, bacteriocins and adhesion inhibitors and have gained prominence as Probiotics. A majority of the strains including L.salivarius were shown to suppress the growth of Aggregatibacter actinomycetemcomitans, Porphyromonos gingivalis and Prevotella intermedia. Probiotic strains included in periodontal dressings at optimal concentration of 108 $\mathrm{CFU} / \mathrm{ml}$ were shown to diminish the number of most frequently isolated periodontal pathogens. ${ }^{5}$

\section{Ozone Therapy ${ }^{6}$}

Ozone is a chemical compound consisting of three oxygen atoms $\mathrm{O}_{3}$ (i.e. triatomic oxygen), a highly energetic form of normal (diatomic) atmospheric oxygen $\left(\mathrm{O}_{2}\right)$. Oxygen/ozone therapy has a long history of research and clinical application with humans. Due to its being an extremely powerful oxidizing agent and a highly effective disinfectant, it is used throughout the world to destroy germs in water treatment installations supplying drinking water.
The German chemist, C.D. Schonbein, first discovered ozone in 1840 . The first medical application was in 1870 when Dr. C. Lender purified blood in test tubes. Medical applications thereafter became widespread throughout Europe and America. Today, more than 114 diseases were listed for treatment with oxygen/ ozone therapy. Interestingly enough, in 1930, a German dentist, Dr. E.A. Fisch, used ozone on a regular basis in his dental practice in Zurich, Switzerland, and published numerous papers on the subject. Dr. Fisch influenced thework of Dr. Erwin Payr, a renowned surgeon. Dr. Payr's work set the stage for mainstream use of oxygen/ozone therapy in medicine.

Ozone is a powerful oxidizer - it effectively kills bacteria, fungi, viruses, and parasites at a dramatically lower concentration than chlorine, with none of the toxic side effects. One molecule of ozone is equal to between 3,000 to 10,000 molecules of chlorine and it kills pathogenic organisms 3,500 times faster!

\section{Application of Ozone Therapy}

Three basic forms of application to oral tissue are applied -1) ozonated water, 2) ozonated olive oil, and

3) oxygen/ozone gas. The rationale behind use of ozone is the safety, efficacy and non- toxicity or side effects.

\section{Ozone therapy}

\begin{tabular}{|l|l|}
\hline \multicolumn{1}{|c|}{ Effects } & \multicolumn{1}{|c|}{ Medical Uses } \\
\hline $\begin{array}{l}\text { Activation of red blood cell metabolism } \\
\text { improved oxygen supply. }\end{array}$ & $\begin{array}{l}\text { Arterial circulatory disorders (peripheral } \\
\text { and cerebral in particular) add. } \\
\text { Complementary in various kinds of } \\
\text { cancer. }\end{array}$ \\
$\begin{array}{l}\text { Activation of immune cells the body releases } \\
\text { its own vital cytokines, such as interferon's and } \\
\text { interleukins. }\end{array}$ & $\begin{array}{l}\text { Revitalization. } \\
\text { General immune weakness. }\end{array}$ \\
\hline $\begin{array}{l}\text { Iincrease and activation of the body's own } \\
\text { antioxidants and radical scavengers }\end{array}$ & $\begin{array}{l}\text { Inflammatory processes, e.g. arthritis, } \\
\text { reactivated arthritis, vascular conditions; } \\
\text { age related processes }\end{array}$ \\
\hline
\end{tabular}

\section{Periodontal Vaccine ${ }^{7}$}

The primary role of any periodontal vaccine would be to eradicate the global periodontal disease burden with the ultimate purpose of lowering periodontal disease associated morbidity in humans. As an innovative strategy, vaccines using cross-reactive immunodominant epitopes as antigenic molecules in an attempt to stimulate antigen-specific regulatory T-cells (Tregs, CD4+, CD25+, FoxP3+), secreting IL-10 and TGF- $\beta$, may provide new clues for periodontal disease prevention, through the induction of either immune tolerance or an effector function. 
Periodontal disease as a multifactorial and polymicrobial disease requires a sophisticated vaccine design regimen targeting multiple pathogenic species. Vaccine regimens including the commonly shared antigens by selected periodontopathogenic species would be considered an innovative strategy.

\section{Photodynamic Therapy ${ }^{8}$}

Photodynamic therapy is based on the principle that a photoactivatable substance (the photosensitizer) binds to the target cell and can be activated by light of a suitable wavelength. During this process, free radicals are formed (among them singlet oxygen), which then produce an effect that is toxic to the cell.

\section{Mechanism of Action}

Briefly, upon illumination, the photosensitizer is excited from the ground state to the triplet state. The longer life time of the triplet state enables the interaction of the excited photosensitizer with the surrounding molecules, and it is generally accepted that the generation of the cytotoxic species takes place during PDT while in this state only. The cytotoxic product, usually $\mathrm{O} 2$ cannot migrate $>0.02 \mathrm{~mm}$ after its formation, thus making it ideal for the local application of PDT without endangering distant molecules, cells, or organs.

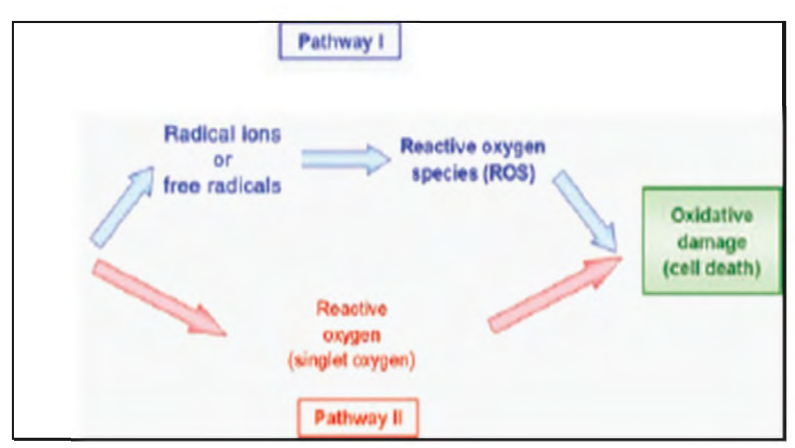

Fig1: Mechanism of Action

\section{Advantages}

PDT is also beneficial during the maintenance of periodontal therapy because it may act on the biofilm and eliminate the need for the removal of additional root substance by mechanical retreatment. Thus, the patient may experience less dentinal hypersensitivity.

This therapy also serves as an adjunct to mechanical therapy in sites with difficult access.

\section{Adverse effects}

PDT has the potential of phototoxic and photo-allergic unwanted side effects

$\checkmark$ There may be impairment of benign oral flora which may lead to an overgrowth of a single resistant species

$\square$ Histologically scar formation is evident

$\checkmark$ Has the potential of promoting genotoxic effects

\section{Gene Therapy ${ }^{9}$}

Application of growth factors or soluble forms of cytokine receptors by gene transfer provides a greater sustainability than that of single protein application. Gene therapy may achieve greater bioavailability of growth factors within periodontal wounds, which may provide greater regenerative potential.

\section{Gene delivery methods}

Genetic engineering approaches generally consist of two modalities: in vivo and ex vivo gene delivery. In the former, gene constructs, such as expression plasmid DNA or a viral particle, are physically entrapped within a scaffold or matrix. When the scaffold containing the gene constructs is implanted into the tissue defect, the host cells migrate into the implant, take up the gene constructs and start to produce the encoded protein.

In the latter approach, cultured cells are transfected (in Nonviral delivery systems) or transduced (in viral delivery systems) with gene constructs in vitro before they are transplanted into the tissue defect.

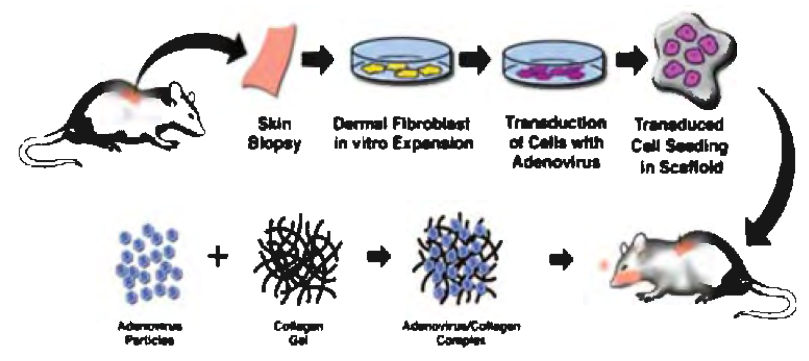

Figure 2. Gene delivery approaches for periodontal tissue engineering. (A) Ex vivo gene delivery. (B) The in vivo gene transfer

\section{Gene Activated Matrix (GAM)}

It has been produced based on the principle of combination of naked DNA with a biodegradable carrier. 


\section{Limitation}

- Difficulties in the preparation of collegen based GAMs and the enormous amount of plasmid required may limit its clinical use.

- Gene therapy studies utilizing bone morphogenetic proteins (BMPs) have also been performed and bone morpbogenetic protein-7 (BMP-7) transduced fibroblasts were used to stimulate repair of alveolar bone wounds and represent the first successful evidence of periodontal tissue engineering employing ex vivo gene transfer of bone morphogenetic proteins.

\section{Advantages}

- Safer and more cost-effective than cell-based therapies.

- Mimic the complex natural process of periodontal tissue formation, because multiple genes, and multiple factors, can be delivered within the bone defect

\section{RNA Interference ${ }^{10}$}

The RNA-mediated silencing process is defined as RNAi, a discovery for which Fire and Mellow received the 2006 Nobel Prize.

RNAi works through small RNAs of approximately 20 to 30 nucleotides that guide the degradation of complementary or semi complementary molecules of messenger RNAs (posttranscriptional gene silencing) or interfere with the expression of certnin genes at the promoter level (transcriptional gene silencing).

\section{RVAl in periodontal regeneration}

Through the silencing of genes that negatively control cell proliferation and cell differentiation or genes that induce inflammation or apoptosis, RNAi may favor tissue regeneration.

Tumor necrosis factor-a-targeted siRNA can suppress osteolysis induced by metal particles in a murine calvaria model, opening the way to the application of RNAi in orthopedic and dental implant therapy.

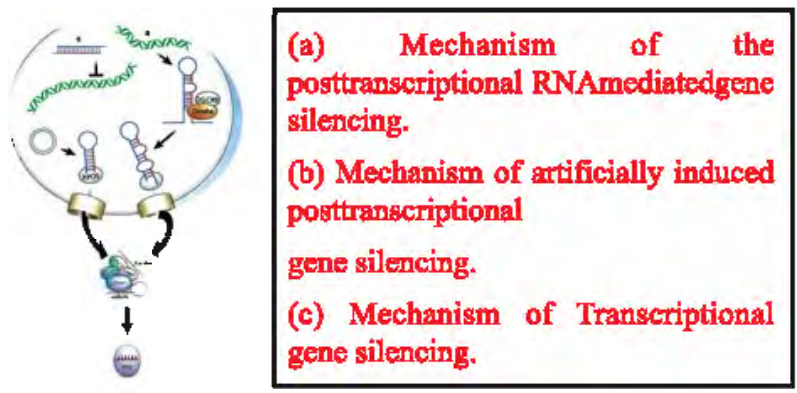

\section{Nantechnology 11}

"The ecience and technology of diagnosing treating and preventing disease and traumatic injury of relieving pain, and of preserving and improving human health, through the use of nanoscale structured materials, biotechnology and genetic engineering and eventually complex molecular machine system and Nanorobots".

\section{Nanorobotics in Dentistry}

Nenorobots induce oral analgesia, Desensitize tooth, manipulate the tissue to re-aligh and straighten irregular set of teeth and to improve durability of teeth.

\section{Nanotechnology in Periodontics}

\section{Mechanism of action}

Functions may be controlled by an onboard nanocomputer execuling programmed instructions in response to local sensor stimuli. Alternatively, the dentist mey issue strategic instruetions by transmitting his orders directly to in vivo nanorobots via acoustic signals (e.g. ultrasound) or by other means.

\section{Treatment Opportunitles in Periodontics}

$\square$ Tooth Repair

$\square$ Hypersensitivity Cure

$\square$ Nanorobotic Dentifrice (Dentifrobots)

\section{Waterlase $^{12}$}

Erbium-Chromium doped: Yittrium-SeleniumGallium-Gamet (Er, Cr: YSGG) laser is commercially available as Waterlase . It uses a patented combination of laser energy and water by a process called Hydro photonics, to perform a wide range of dental procedures.

\section{Drawbacks}

As the Waterlase is quite expensive, it may not make it into many smaller dental practices.

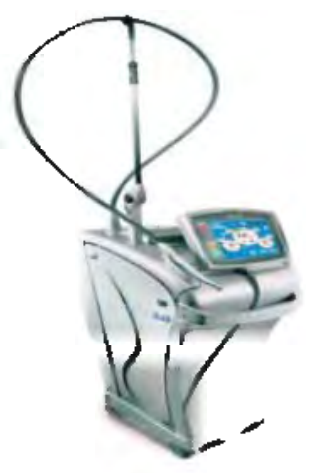




\section{Perioprotect}

PerioProtect is a comprehensive method that is customized for individual patients to help manage biofilms,growing in the spaces or pockets between teeth and gum tissue. The overall goal of the Perio Protect Method is to manage oral biofilm with minimally invasive dentistry for lasting oral health.

The Method is a combination of treatments, including a non-invasive chemical debriding therapy used in conjunction with traditional mechanical debriding procedures. The chemical therapy involves a tray delivery of doctor-prescribed solutions to chemically debride biofilm from the periodontal pocket and alter the pocket's microbiological environment to disrupt biofilm growth.

\section{Tri-Immunophasic Therapy ${ }^{13}$}

William Hoisington has developed a new technique for treatment that allegedly tackles the issue of periodontal disease in an entirely new way.

\section{Development of TIP and BOST}

TIP and BOST have been elaborated and tested over the past several years on over 2,500 patients with remarkably consistent success in saving teeth thought lost, and limiting anaerobic bacteria generated inflammation to an acceptable minimum (Hoisington et al 2005).

\section{Technique}

First DNA test should be done to identify the bacteria to choose the antibiotic of choice. Gingiva is intentionally stretched with the rounded back of curettes in a three stage process. The tissue becomes more stretched as the instruments advance down to the bone level.

Here the curettes are inverted to allow the rounded tip of the curettes to plasty the surface of the bone and remove any attached granulation tissue or degenerated attachment. The goal is a smooth, regular bone surface and fresh bleeding to flush out bacteria and toxins from the porosities. right, where the immune reaction of inflammation can change into one of regeneration. This type of healing happens with the help of the stem cells from the periodontal ligament.The clot that is firmly attached to the clean bone serves as a scaffold. The stem cells can move along it and up the root surfaces at the rate of $0.5 \mathrm{~mm}$ per day for eight days and thicken the layer on the clot. To permit this activity it is also important to keep the epithelial attachment away from the roots. This is done with the oral hygiene technique that keeps the pocket open and also inhibits the reformation of the sticky layer.
As healing time increases, the pockets gradually fill in from the bottom with very dense, partially mineralised connective tissue in about four to six weeks, and finally will become acellular.

\section{Conclusion}

Clinicians should continue to develop and enhance their diagnostic skills, assess factors that affect diagnosis and prognosis, formulate a comprehensive treatment plan, render appropriate treatment, evaluate the outcome and determine when periodontal care is indicated.

\section{References}

1.Wim Teughels, Mark Van Essche, Isabelle Sliepen \& Marc Quirynen. Probiotics and oral healthcare. Periodontology 2000 2008;48:111-147.

2. Harish $\mathrm{K}$, Varghese T. Probiotics in humans evidence based review. Calicut Medical Journal 2006;4(4):e3.

3. Suvarna VC and Boby VU. Probiotics in human health: A current Assessment. Current Science 2005;88(11):1744-1748.

4. Rajiv Saini, Santosh Saini, Sugandha. Probiotics: The Health Boosters. Journal of Cutaneous and Aesthetic Surgery 2009;2(2):112.

5. Deepa D, Mehta DS. Is the role of probiotics friendly in the treatment of periodontal diseases!! Journal of Indian Society of Periodontology 2009;13(1):30-31.

6. Mollica \& Harris Integrating oxygen/ ozone therapy into your practice.

7. Choi1 \& J. Seymour Vaccines against periodontitis: a forward-looking review J Periodontal Implant Sci 2010;40:153-163.

8. Malik R, Manocha A, Suresh DK. Photodynamic Therapy- A Strategic Review Indian J Dent 21(2):2010.

9. Buch A \& Choksi D Clinical Applications Of Stem Cells In Dentistry.

10. Grover V, Malhotra R, Kapoor A, Verma N, Sahota JK Future of Periodontal Regeneration J Oral Health Comm Dent 2010;4(Spl)38-47.

11. S. Hiremath SS, Anegundi RT. Nanorobotics In Denstry http://www.dharwadhubli.com - dharwadhubli. com

12. Nitin Tomer New Innovative Technology: Waterlase in Periodontics People's Journal of Scientific Research Vol.3(1), Jan 2010.

13. William Hoisington New developments in perio: Tri-Immuno-Phasic therapy. Preventive Dentistry Volume 1 issue 2.2006. 